\title{
LA PAROLE OPERANTE COMME SPECIFICATION DE L'INTENTIONNALITE MOTRICE CHEZ MERLEAU-PONTY
}

JAN HALÁK* fondamentalement incarné et mis en œuvre par des actes d'un sujet situé dans un environnement naturel et culturel. Plus précisément, mon objectif est de montrer que le corps est impliqué dans la cognition en tant qu'opérateur de la structuration phénoménale de l'environnement même au niveau de l'expérience linguistique, rationnelle et abstraite.

On sait bien que Merleau-Ponty a inspiré un nombre important de théoriciens de la cognition incarnée. En particulier, les enactivistes comme Francisco Varela, Evan Thompson, ou Shaun Gallagher se sont appuyés sur les travaux de Merleau-Ponty en ce qui concerne l'incarnation et la perception. Ces chercheurs ont apprécié que Merleau-Ponty, étant un phénoménologue, était aussi très ouvert à la recherche scientifique, ce qui faisait de lui une sorte de chercheur en science cognitive avant la lettre. ${ }^{1}$ Comme le souligne Vörös, par exemple, la phénoménologie de Merleau-Ponty est particulièrement bien adaptée pour un dialogue avec les recherches cognitives-scientifiques actuelles, car elle ne commence pas par la réflexion pure mais, au contraire, s'appuie sur
Mon but dans ce texte est de décrire comment Merleau-Ponty interprète la cognition d'ordre supérieur comme un processus

Keywords: Merleau-Ponty; phenomenology; embodied cognition; higher-order cognition; moto intentionality; philosophy of language; mathematical reasoning.

\section{Introduction}

* Palacký University Olomouc, Faculty of Arts, Department of Philosophy, Kř́žkovského 12, Olomouc, 771 48, Czech Republic, Email: jan.halak@upol.cz. ORCID: 0000-0001-8482-7168

1 Voir par exemple S. Gallagher, "Merleau-Ponty's Phenomenology of Perception », Topoi, 29(2), 2010, 183-185; " Cognitive Science », in R. Disprose, J. Reynolds (Eds.), Merleau-Ponty: Key Concepts, Acumen, 2014, 207-217. 
des d'études psychologiques, psychiatriques, neurologiques, ou biologiques. ${ }^{2}$

Merleau-Ponty explique son rapport à la recherche scientifique d'une manière encore plus précise dans son cours de 1953. II rejette " l'empirisme et la science purement causale ", mais souligne que la science fournit des " faits dont la philosophie doit, [et] peut, tirer partie $»^{3}$. Pour lui, la réflexion philosophique et l'étude des faits scientifiques ne sont pas hétérogènes, et il y a même " nécessité d'introduire les faits " ${ }^{4}$ scientifiques dans la philosophie. Pour lui, le rôle des faits tels que les descriptions neurologiques et psychiatriques de l'apraxie " n'est pas de suppléer la réflexion " philosophique, mais de « nous fournir des variantes de notre expérience qui nous éveillent à ce qu'elle contient $\nu^{5}$ et qui ne sont pas accessibles autrement. Dans le cadre philosophique de Merleau-Ponty, les faits positifs tel que les déterminants physiques-causaux décrits par la pathologie nerveuse sont donc toujours pris en compte, mais jamais considérés comme explicatifs. Sur ce point, son interprétation du sujet incarné et de la cognition se situe

2 S. Vörös, « Mind Embodied, Mind Bodified. Merleau-Ponty and the Enactive Turn in Mind Sciences ". Etudes phénoménologiques - Phenomenological Studies, 4/2020, 7, 13.

3 M. Merleau-Ponty, Le monde sensible et le monde de l'expression, Metis Presses, 2011, 154.

4 Ibid., 153.

5 Ibid.; cf. ibid., 93.

6 Toutefois, plusieurs commentateurs se sont aussi opposés à un tel rapprochement entre MerleauPonty et la science cognitive. Voir, par exemple, R. M. Muller, " Merleau-Ponty and the radical sciences of mind ", in Synthese, 198/2021, 22432277; C. Pollard, « Merleau-Ponty and embodied assez proche des théories relationnelles et dynamiques issues de l'enactivisme autopoïétique ou le cadre conceptuel $4 \mathrm{E} .{ }^{6}$

Cependant, la réception actuelle de Merleau-Ponty par les cognitivistes semble attaindre ses limites avec le thème de la cognition supérieure. Les théoriciens de la cognition incarnée ne discutent généralement pas des idées de Merleau-Ponty sur le rôle de l'intentionnalité motrice dans la cognition supérieure. II est pourtant clair que pour Merleau-Ponty, le corps et la motricité corporelle jouent un rôle très important dans la cognition supérieure. MerleauPonty note, par exemple, que l'on «ne pense pas sans le corps transfiguré, porteur de significations, qui est le schéma corporel ". ${ }^{7}$ Le corps en ce sens est, pour Merleau-Ponty, " le porteur d'un nombre indéfini de systèmes symboliques » car ces systèmes " s'effondrent si le corps cesse d'en ponctuer l'exercice et de les installer dans le monde et dans notre vie ${ }^{8} .^{8}$ Pour MerleauPonty, le rôle du corps et de sa mobilité dans la cognition d'ordre supérieur est clairement fondateur. cognitive science ", in Discipline Filosofiche, 24(2)/ 2014, 67-90. Cependant, Pollard (op. cit., 89) finit son article par reconnaître que le mode d'enquête "transcendantal-philosophique " de Merleau-Ponty n'exclut pas les descriptions empiriques et scientifiques. Voir aussi Vörös, op. cit., 7, 14 ; S. Gallagher, "Rethinking Nature: Phenomenology and a Non-reductionist Cognitive Science ", in Australasian Philosophical Review, 2 (2) / 2018, 125-137.

7 M. Merleau-Ponty, Le monde sensible et le monde de l'expression, Metis Presses, 2011, 162.

8 M. Merleau-Ponty, Résumés de cours, Gallimard, 1968, 18. 
Corrélativement, dans le domaine de la recherche sur la cognition incarnée, peu attention a été accordée aux sujets qui sont devenus important dans la recherche plus spécialisée de Merleau-Ponty au cours des vingt dernières années. En particulier, les travaux de Merleau-Ponty sur le langage datant de l'après-guerre, y compris ses premiers cours au Collège de France, peuvent approfondir de manière considérable notre compréhension de l'interprétation classique de l'incarnation et de l'expérience perceptive développées dans la Phénoménologie de la perception et la Structure du comportement.

Donc, bien que mon but ici ne soit pas d'analyser la relation positive et fructueuse de Merleau-Ponty à la recherche empirique, ${ }^{9}$ mon ambition est de fournir une illustration concrète de l'approche intégrative et interdisciplinaire de Merleau-Ponty. En outre, j'aimerais clarifier les affirmations de Merleau-Ponty sur la relation entre la corporéité et la cognition supérieure en abordant trois sujets principaux. Tout d'abord, je vais décrire comment, s'appuyant sur une étude concrète issue de neuropathologie, MerleauPonty développe le concept d'intentionnalité motrice comme une capacité de différenciation dynamique des figures perceptives. Ensuite, je vais expliquer comment Merleau-Ponty s'approprie des éléments importants de la linguistique structuraliste de Saussure et l'idée de langage comme un

9 Sur ce point, voir surtout J. Reynolds, « MerleauPonty's Gordian knot: Transcendental phenomenology, science, and naturalism ", in Continental Philosophy Review, 50(1) / 2017, 81-104 ; K. Romdenh-Romluc, "Science in Merleau- Ponty's phenomenology: From the early work to the later philosophy ", in D. Zahavi (Ed.), Oxford Handbook of the History of Phenomenology, système de différences entre les signes et comment il relie cette idée à son interprétation de l'intentionnalité motrice. Finalement, je vais brièvement expliquer comment Merleau-Ponty interprète la pensée mathématique, qu'il comprend comme un cas particulier de la parole parlante ou opérante. Cela devrait permettre d'esquisser une théorie merleau-pontyenne de la cognition supérieure comme un processus fondamentalement incarné et supporté par des activités intentionnelles d'un corps situé et mobile.

\section{Intentionnalité motrice comme différenciation de l'environnement phénoménal}

L'exposé de Merleau-Ponty sur l'intentionnalité motrice se fonde principalement sur le cas bien connu de Schneider, un ancien combattant victime d'une lésion cérébrale. Selon l'interprétation originale de Gelb et Goldstein, ${ }^{10}$ Schneider souffrait d'agnosie visuelle ou d'une "cécité psychique ", c'està-dire d'une incapacité de proprement articuler les formes perceptives (Gestalten). Schneider ne reconnaissait généralement pas les objets par la seule vision, ce qui affectait également ses expériences tactiles et motrices. Plus important encore, Schneider avait des difficultés à effectuer des mouvements dits « abstraits », qui n'étaient pas sollicités par son environnement sensorimoteur immédiat.

Oxford University Press, 340-359. (2014, 89); S. Matherne, « Merleau-Ponty on abstract thought in mathematics and natural science ", in European Journal of Philosophy, 26(2) / 2018, 780797.

${ }^{10}$ A. Gelb \& K. Goldstein, Psychologische Analysen hirnpathologischer Fälle. Johann Ambrosius Barth, 1920. 
Selon Gelb et Goldstein, les difficultés de Schneider provoquaient une perte de la fonction de représentation, attitude catégoriale, ou de la capacité de projeter spontanément ses intentions dans l'environnement.

Schneider et l'interprétation de Gelb et Goldstein sont encore discutés aujourd'hui dans la littérature. ${ }^{11}$ Quant à Merleau-Ponty, il a des doutes sur l'explication de Gelb et Goldstein. Il affirme, par exemple, que l'expérience visuelle $n^{\prime}$ est jamais simplement ajoutée aux expériences tactiles ou motrices, et ne peut donc pas être simplement soustraite de la perception totale du patient, comme l'affirment Gelb et Goldstein. ${ }^{12}$ Selon Merleau-Ponty, Schneider ne manquait pas simplement certaines parties de son expérience (comme les sensations visuelles) ou une faculté épistémologique spécifique (comme la fonction de projection ou de représentation). La déficience semble plutôt se définir par le fait que la structure globale de l'expérience de Schneider est moins différenciée, organisée d'une manière moins complexe.

Cette interprétation peut être élaborée par une analyse des performances compensatoires de Schneider. Par exemple, Schneider ne pouvait pas déterminer immédiatement la position d'un point de son corps qui

11 Voir G. Goldenberg, « Goldstein and Gelb’s Case Schn.: A classic case in neuropsychology? ", in Ch. Code, C.-W. Wallesch, Y. Joanette, A. R. Lecours (eds), Classical Cases in Neuropsychology, vol. II, Psychology Press, 2012, 281-299. En rapport avec Merleau-Ponty, voir surtout R. T. Jensen, "Motor intentionality and the case of Schneider ", in Phenomenology and the Cognitive Sciences, 8(3) / 2009 ; T. Mooney, « Plasticity, motor intentionality and concrete movement in Merleau-Ponty ", Continental Philosophy Review, 44(4) / 2011, 359-381 ; G. B. Jackson, « Maurice Merleau-Ponty's concept of motor intentionality: Unifying two kinds of bodily était touché, mais il pouvait le localiser en mettant tout son corps en mouvement et en spécifiant progressivement la localisation du point par des mouvements exploratoires supplémentaires. ${ }^{13}$ Ce qui est encore plus intéressant, Schneider pouvait accomplir des tâches motrices autrement impossibles en s'appuyant sur des structures culturelles sédimentées, telles que le langage ou les séries arithmétiques, qu'il utilisait comme des "scénarios" pour l'action. Par exemple, il analysait des indices perceptifs en termes de structures préétablies fournies par le langage, puis subsumait les premiers sous les seconds à l'aide d'inférences logiques.

Selon Merleau-Ponty, les actions compensatoires de Schneider doivent être comprises comme des tentatives de développer la structuration de son environnement phénoménal, qui compensent la détérioration de ses capacités motrices et son retour à une relation au monde plus globale. Par conséquent, la fonction originale de l'intentionnalité motrice, qui est altérée chez Schneider, ne doit pas être comprise comme un complexe d'états ou de fonctions liés au contenu ou à la représentation, mais plutôt comme un processus de structuration. ${ }^{14}$ De ce point de vue, percevoir signifie utiliser activement ses capacités corporelles pour articuler des figures

agency ", European Journal of Philosophy, 26(2) / 2017, 763-779.

12 Voir M. Merleau-Ponty, Phénoménologie de la perception, Gallimard, 1945, 150-151; Le monde sensible et le monde de l'expression, Metis Presses, 2011, 141-142.

${ }_{13} \mathrm{M}$. Merleau-Ponty, Phénoménologie de la perception, Gallimard, 1945, 137; Le monde sensible et le monde de l'expression, Metis Presses, 2011, 141.

${ }^{14}$ Voir R. T. Jensen, " Motor intentionality and the case of Schneider ", in Phenomenology and the Cognitive Sciences, 8(3) / 2009, 386-387. 
perceptives. Comme l'écrit Merleau-Ponty en se référant à Grünbaum, ${ }^{15}$ l'intentionnalité motrice implique « la capacité de différenciation motrice du schéma corporel dynamique » qui correspond à " une certaine manière de mettre en forme ou de structurer l'entourage ${ }^{16}{ }^{16}$

\section{Le langage comme système diacritique}

Or, les écrits de Merleau-Ponty de l'après-guerre permettent de montrer une stricte continuité entre son interprétation de l'intentionnalité motrice et ses vues sur le langage. Comme Merleau-Ponty l'explique dans une note de travail de 1953, la théorie saussurienne du langage, qui définit le signe par sa valeur "diacritique ", et les travaux plus récents de Goldstein ${ }^{17}$ rejoignent "l'idée profonde de la pathologie nerveuse » selon laquelle une maladie n'est pas une "soustraction " de certaines parties de notre expérience, mais une " dédifférenciation " de l'expérience comme un tout. ${ }^{18}$ La continuité entre l'expérience perçue et parlée que Merleau-Ponty ainsi retrouve, lui permet à son tour d'argumenter en faveur d'un rôle constitutif du corps et

${ }^{15}$ A. A. Grünbaum, "Aphasie und Motorik », in Zeitschrift für die gesamte Neurologie und Psychiatrie, 130(1) / 1930, 397-398.

${ }^{16} \mathrm{M}$. Merleau-Ponty, Phénoménologie de la perception, Gallimard, 1945, 177-178 et 146.

17 Voir K. Godstein, Language and language disturbances; aphasic symptom complexes and their significance for medicine and theory of language, Grune and Stratton, 1948.

$18 \mathrm{M}$. Merleau-Ponty, Le problème de la parole, Metis Presses, 2020, 123; voir aussi ibid., 119120. de la motricité aux niveaux "supérieurs » de la cognition.

Comme on le sait, après avoir publié la Phénoménologie de la perception en 1945, Merleau-Ponty entreprend une étude approfondie de la linguistique de Saussure. Vers 1951 , la parole et le langage deviennent les sujets centraux dans son projet de livre La Prose du monde. Ses trois premières cours au Collège de France (1953-1954) sont consacrées à l'expression culturelle, à I'usage littéraire du langage et au problème de la parole. Dans ces travaux, MerleauPonty développe son interprétation du langage et souligne à quel point il imprègne toute notre vie, y compris nos expériences corporelles.

À cette époque, Merleau-Ponty adhère à l'idée de Saussure selon laquelle " dans la langue, il n'y a que des différences sans termes positifs ${ }^{19}$. De ce point de vue, la signification d'un signe est déterminée non pas par ce qu'il contient positivement, mais par sa différence avec les autres signes. En d'autres termes, la signification des signes est déterminée par leur valeur diacritique. ${ }^{20}$ Merleau-Ponty apprécie le cadre structurel de Saussure car il lui permet de critiquer l'idée objectiviste selon laquelle les signes possèdent une unité positive

19 F. de Saussure, Cours de linguistique générale, Payot, 1971, 166; cité par M. Merleau-Ponty, La prose du monde, Gallimard, 1969, 45.

20 Cf. M. Merleau-Ponty, Résumés de cours, Gallimard, 1968, 33. Selon toute évidence, Merlau-Ponty reprend l'idée de " diacritique » non pas de Saussure lui-même, mais de l'ouvrage d'un de ses successeurs, R. Jakobson, (voir Kindersprache, Aphasie, und allgemeine Lautgesetze, De Gruyter Mouton, 1969). 
et se réfèrent à des référents univoques ou des objets en soi. Si les signes n'ont de sens que par référence à d'autres signes, et non aux objets, ils ne signifient pas en vertu de leur relation à quelque chose qui existerait en dehors d'un système signifiant.

Cependant, contrairement à l'interprétation traditionnelle du structuralisme, Merleau-Ponty interprète le système diacritique du langage comme fondamentalement ouvert et dynamique. Pour lui, les actes de parole individuels ne sont pas simplement subordonnés au système linguistique, mais le remodèlent dynamiquement. Merleau-Ponty soutient que Saussure luimême mettait en cause la distinction entre les actes de parole contingents et leur valeur générale dans le système de la langue. $^{21}$ La parole, écrit Merleau-Ponty, " ne réalise pas seulement les possibilités inscrites dans la langue, ... elle modifie et soutient la langue tout autant qu'elle est portée par elle ${ }^{22}$. Plutôt que d'être une simple enveloppe externe et un instrument de la pensée, la parole est pour MerleauPonty un véhicule intentionnel permettant de se mettre en rapport avec un type particulier de signification et de la saisir. Cette approche implique que le système linguistique et, par conséquent, conceptuel est soumis à un développement par la manière dont les sujets parlants s'approprient ou produisent, à travers leurs actes de parole

${ }^{21}$ M. Merleau-Ponty, Résumés de cours, Gallimard, 1968, 33.

22 Ibid.

${ }^{23}$ Voir sur ce point A. P. Foultier, "MerleauPonty's Encounter with Saussure's Linguistics: Misreading, Reinterpretation or Prolongation? ", in Chiasmi International, 15 / 2013, 129-150 ; B. Stawarska, "Uncanny Errors, Productive Contresens. Merleau-Ponty's Phenomenolo- individuels, des variations linguistiques et dont ils les intègrent dans la totalité des normes communicatives préétablies.

Comme on le sait, l'accent mis par Merleau-Ponty sur le rôle de la parole et des actes linguistiques individuels a souvent été perçu comme une incompréhension de la théorie de Saussure. ${ }^{23}$ Selon la lecture structuraliste $"$ doctrinale ${ }^{24}$, la linguistique structurale sépare strictement les aspects systématiques du langage des aspects contingents, et par conséquent la synchronie de la diachronie. De ce point de vue, la parole ne peut être comprise linguistiquement que dans le contexte du système linguistique, car elle implique potentiellement des déformations non systématiques et accidentelles. Les énoncés individuels provoqueraient des changements diachroniques du système dans la mesure où ils sont précisément non systématiques, et ne refléteraient pas la volonté d'un sujet de transmettre un sens spécifique. Définie en ces termes, la linguistique structurale ne peut étudier ni l'innovation linguistique ni l'activité innovatrice au niveau de la pensée abstraite et conceptuelle.

Toutefois, tenant compte du contexte éditorial de l'édition de 1916 du Cours de linguistique générale et des écrits originaux de Saussure, les affirmations sur l'incompatibilité des travaux de Merleau-Ponty avec une compréhension structurale du langage

gical Appropriation of F. de Saussure's General Linguistics ", in Chiasmi International, 15 / 2013, 151-165.

${ }^{24}$ Voir les travaux de B. Stawarska, Saussure's Philosophy of Language as Phenomenology: Undoing the Doctrine of the Course in General Linguistics, Oxford University Press, 2015; Saussure's Linguistics, Structuralism, and Phenomenology, Palgrave Macmillan, 2020. 
se révèlent peu convaincantes. Bien que Merleau-Ponty n'ait pas eu accès à d'autres écrits que le Cours, il était particulièrement attentif à ce qu'il percevait comme des ambiguïtés conceptuelles du texte. II met précisément l'accent sur les aspects de la pensée de Saussure qui ont été marginalisés par les éditeurs de l'édition de 1916 du Cours et les interprétations traditionnelles du structuralisme et qui sont maintenant redécouverts au sein de la recherche saussurienne. Comme le souligne aujourd'hui Stawarska, la distinction de Saussure entre langue et parole " est de degré et non de nature, et relative plutôt qu'absolue $"{ }^{25}$ Ainsi, selon Saussure, les actes de parole individuels ne sont pas déterminés unilatéralement par les valeurs systématiques d'une langue, comme le prétend l'approche structuraliste traditionnelle. Au contraire, les deux ordres sont interdépendants et incorporent constamment les différenciations de l'autre. ${ }^{26}$ Dans cette optique, la langue n'est pas un système fermé sur soi, mais un équilibre de stabilité et de changement qui est ouvert aux actes des locuteurs individuels. $^{27}$

Comme l'explique précisément Merleau-Ponty, la parole expressive ne communique donc ni les expériences subjectives indépendantes du langage ni des valeurs inscrites préalablement dans une langue supra-individuelle, mais articule plutôt "l'excès de ce que nous vivons sur ce qui a

25 B. Stawarska, Saussure's Linguistics, Structuralism, and Phenomenology. Palgrave Macmillan, 2020, 84.

26 Cf. ibid., 82, 118.

27 Cf. ibid., Chapitre 13, qui analyse l'apport de Merleau-Ponty sur ce point.

28 M. Merleau-Ponty, La prose du monde, Gallimard, 1969, 158-159 et 155. déjà été dit "; par conséquent, " le contact prétendu avec [les choses] n'est pas au début de la langue, mais au bout de son effort $"{ }^{28}$ Cela signifie, en bref, que les objets n'existent pas indépendamment de nos relations avec eux, mais qu'ils apparaissent plutôt en fonction de notre capacité à articuler leur structure phénoménale à travers les systèmes "diacritiques » dont nous disposons collectivement. La perception, comme nous l'avons vu, est un tel système dynamique de différenciation fondé sur notre motricité ${ }^{29}$ - et le langage en est un autre, fondé sur la parole opérante. Au sein de ces systèmes diacritiques entrelacés, nous ne nous rapportons jamais linguistiquement à de simples référents, mais plutôt à ce qui reste à dire, dans notre situation totale, par rapport à ce qui a déjà été articulé dans notre langue et notre culture.

Par conséquent, Merleau-Ponty soutient que la pensée abstraite articulée conceptuellement, qui était censée être absente dans le cas de Schneider, est le résultat du processus de " différenciation parallèle du langage et de la situation $" .^{30}$ Pour Merleau-Ponty, la fonction "supérieure " telle que l'articulation linguistique de la pensée n'est donc pas simplement ajoutée à une fonction " inférieure " telle que l'action sensorimotrice, mais se constitue plutôt à travers une structuration plus fine de ce qui reste seulement globalement organisé, ou "polymorphe ", dans notre expérience

${ }^{29}$ Merleau-Ponty parle même d'une « perception diacritique ", se référant à la linguistique de Saussure. Voir Le monde sensible et le monde de l'expression, Metis Presses, 2011, 203-204.

${ }^{30} \mathrm{M}$. Merleau-Ponty, Le problème de la parole, Metis Presses, 2020, 121. 
sensorimotrice. Inversement, les pathologies du langage correspondent à un retour à une organisation plus amorphe de l'expérience. ${ }^{31}$ Pour Merleau-Ponty, il y a donc une stricte continuité entre le niveau sensorimoteur et le niveau linguistique, qui est fondée sur une différenciation phénoménale accomplie par le corps moteur et la parole comme un instrument de sublimation de nos intentions motrices.

\section{Rationalité incarnée : exemples de géométrie et algèbre}

Pour illustrer plus concrètement l'approche de Merleau-Ponty de la parole comme véhicule à travers lequel l'intentionnalité motrice est prolongée et sublimée, je vais maintenant aborder brièvement un type de cognition que les théories intellectuelles présument représenter le sommet de la rationalité et donc de la désincarnation : la pensée mathématique.

Ici encore, Merleau-Ponty plaide en faveur d'un rôle fortement constitutif du corps. Dans la Phénoménologie de la perception, il affirme par exemple que " le sujet de la géométrie est un sujet moteur $»{ }^{32}$ Dans la Prose du monde, il soutient de la

31 lbid.

32 M. Merleau-Ponty, Phénoménologie de la perception, Gallimard, 1945, 443.

${ }^{33}$ Voir surtout M. Merleau-Ponty, La prose du monde, Gallimard, 1969, 149-151, 161-181.

34 Sur ce point, l'approche de Merleau-Ponty préfigure des aspects importants de la recherche contemporaine sur les mathématiques. Concernant la géométrie, voir par example M. Hohol, Foundations of Geometric Cognition, Routledge, 2020, Chapitre 4. même manière qu'il n'y a pas de pensée désincarnée dans l'algèbre. ${ }^{33}$ Dans les deux cas, Merleau-Ponty maintient que la configuration des signes mathématiques, tels que les diagrammes géométriques et les symboles mathématiques, joue un rôle fondamental dans notre accès aux idées mathématiques ${ }^{34}$ et que cette configuration est un phénomène perceptif qui est profondément lié à l'action corporelle. Alors que, pour les cadres théoriques intellectualistes, c'est une question d'indifférence comment, parmi les diverses manières possibles, nous nous représentons un objet mathématique, Merleau-Ponty soutient que cela n'est vrai qu'en dessous d'un certain seuil, et non absolument. Dans la mesure où la réalisation d'un acte de compréhension mathématique requiert des réorganisations structurelles $\mathrm{d}^{\prime}$ acquisitions culturelles antérieures, la pensée en géométrie et en algèbre est incarnée pour Merleau-Ponty.

Sur ce point, il est important de noter que Merleau-Ponty reconnaît l'argument de Gurwitsch ${ }^{35}$ contre Wertheimer ${ }^{36}$ et la psychologie gestaltiste en général, selon lequel une entité mathématique telle qu'un triangle ne peut être interprétée comme une gestalt perceptive, car sa signification ne dépend pas directement du contexte

35 A. Gurwitsch, « Some Aspects and Developments of Gestalt Psychology ", in F. Kersten (Ed.), The Collected Works of Aron Gurwitsch (1901-1973): Volume II: Studies in Phenomenology and Psychology, Springer Science \& Business Media, 2009, 58-61.

${ }^{36}$ M. Wertheimer, " The Syllogism and Productive Thinking ", in W. D. Ellis (Ed.), A Source Book of Gestalt Psychology, Kegan Paul, Trench, Trubner \& Company, 1938, 274-282. 
perceptif. ${ }^{37}$ Contrairement à un objet perceptuel concret, un objet mathématique a le pouvoir d'inaugurer un ordre de signification dans lequel les changements phénoménaux sont soit non pertinents, soit ils manifestent le même objet de manière plus complète. Cependant, selon Merleau-Ponty, une signification idéale n'est jamais complètement détachée de son contexte phénoménal. Plutôt, la nature spécifique des signes qui la présentent élève, pour ainsi dire, le seuil au-delà duquel le contexte phénoménal affecte leur signification.

Merleau-Ponty donc refuse également l'explication husserlienne de Gurwitsch, comparable à celle que Gelb and Goldstein ont appliqué au cas de Schneider, selon laquelle un objet abstrait (mathématique ou autre) est le corrélat d'une " attitude catégorielle " et par conséquent détachable du champ phénoménal spatio-temporellement concret. $^{38}$ Merleau-Ponty soutient que la «fonction de représentation " présumée, qui est censée permettre l'attitude " catégorielle ", repose sur un certain fondement, et ne doit pas être séparée des " matériaux » dans lesquels elle se réalise. ${ }^{39}$ Plus précisément, MerleauPonty affirme que l'attitude catégoriale et ses corrélats intentionnels sont est en réalité achevés par la structuration (Gestaltung)

37 M. Merleau-Ponty, Phénoménologie de la perception, Gallimard, 1945, 444.

${ }^{38}$ Voir A. Gurwitsch, "Some Aspects and Developments of Gestalt Psychology ", in F. Kersten (Ed.), The Collected Works of Aron Gurwitsch (1901-1973): Volume II: Studies in Phenomenology and Psychology, Springer Science \& Business Media, 2009, 54-61.

39 M. Merleau-Ponty, Phénoménologie de la perception, Gallimard, 1945, 157.

40 M. Merleau-Ponty, Le problème de la parole, Metis Presses, 2020, 121. active de la relation entre un sujet et le monde appuyée sur des symboles culturelles. ${ }^{40}$ L'attitude catégoriale ne préexiste donc pas comme une fonction épistémologique générale, mais se réalise au sein d'une situation spatio-temporelle concrète et reste à jamais ouverte aux réorganisations ultérieures, ou même à une désintégration.

S'appuyant sur les travaux de Wertheimer, Merleau-Ponty ainsi soutient que l'on ne peut saisir une signification proprement géométrique qu'en se rapportant à une " configuration » d'espace circonscrite par un triangle, par exemple, situé dans l'espace orienté de notre champ visuel, ou dans le champ de notre imagination visuelle. ${ }^{41} \mathrm{De}$ plus, Merleau-Ponty affirme que le système de positions spatiales circonscrites par le triangle est aussi un champ de mouvements possibles pour moi en tant que sujet moteur. Une figure géométrique n'est donc donnée ni comme un fait positif enregistré par les sens, ni comme une essence transcendante saisie par la raison. C'est plutôt une essence concrète, c'est-à-dire une gestalt située dans notre champ perceptif et moteur. Par conséquent, plutôt que d'être une partie du monde, un objet matériel ou idéal, une figure géométrique relève de notre relation au monde. Les propriétés géométriques spécifiques ne nous

41 Voir M. Merleau-Ponty, Phénoménologie de la perception, Gallimard, 1945, 443-448; M. Wertheimer, "The Syllogism and Productive Thinking ", in W. D. Ellis (Ed.), A Source Book of Gestalt Psychology, Kegan Paul, Trench, Trubner \& Company, 1938, 279-80. Voir également l'interprétation de ces passages par $\mathrm{M}$. Hass \& L. Hass, L., "Merleau-Ponty and the Origin of Geometry » in F. Evans (Ed.), Chiasms: Merleau-Ponty's Notion of Flesh, SUNY Press, 2000, 177-187 ; L. Hass, Merleau-Ponty's Philosophy, Indiana University Press, 2008, 150-155. 
sont pas accessibles sur la base d'une simple analyse logique d'un objet géométrique en tant que représentation mentale. Au contraire, on ne parvient à saisir une vérité touchant un objet géométrique tel qu'un triangle qu'en réorganisant notre rapport à lui et en transformant sa configuration phénoménale concrète.

Ainsi, afin de démontrer une vérité géométrique, le géomètre doit intervenir dans la figure du triangle comme le pôle vers lequel ses mouvements sont dirigés ; il " explore » la configuration spatiale que le triangle lui ouvre, il se situe en un point et de là tend vers un autre point ; il étend un côté du triangle, puis trace une ligne à travers le sommet qui est parallèle au côté opposé, et ainsi de suite. ${ }^{42}$ C'est donc en réorganisant une configuration phénoménale concrète, par exemple en l'explorant perceptivement ou en y ajoutant des lignes auxiliaires, que le géomètre arrive à voir et à démontrer pour tout autre géomètre que la somme des angles d'un triangle est égale à deux angles droits.

De même, Merleau-Ponty maintient que le mathématicien n'accède à certaines propriétés algébriques des nombres qu'en structurant la série numérique linéaire d'une manière spécifique. Dans le cas de la formule de Gauss $\Sigma n=(n \div 2) \times(n+1)$, par exemple, la série numérique est structurée de telle sorte que ses membres forment des paires de même valeur. ${ }^{43} L^{\prime}$ 'importance d'une formule mathématique consiste en ce qu'elle

42 M. Merleau-Ponty, Phénoménologie de la perception, Gallimard, 1945, 444-446.

43 Voir surtout M. Merleau-Ponty, La prose $d u$ monde, Gallimard, 1969, 149-151; L'institution, la passivité. Notes de course au Collège de France (1954-1955), Belin, 2003, 95-96; M. Wertheimer, Productive Thinking, Birkhäuser, 2020, 108-142. contracte certaines transformations structurelles de la série de nombres, ce qui articule un aspect mathématique de la série qui ne serait pas disponible autrement. Sans la formule de Gauss, il faudrait compter la somme de la série en procédant d'une manière progressive, car la relation gaussienne n'est pas évidente dans la série donnée comme une série linéaire. Avec la formule, en revanche, l'exécution progressive des additions devient inutile.

En outre, une formule originale comme celle de Gauss a priorité sur toutes les autres formules algébriques correctes qui peuvent en être dérivées par formalisation et qui ont la même validité mathématique. Les formules dérivées ne conservent pas sa " lumière démonstrative " qui est liée à sa configuration structurelle unique. ${ }^{44}$ Tandis que la formule originale permet d'effectuer à nouveau les étapes exploratoires qui y sont contractées, les formules dérivées ne rendent pas directement accessible une telle réactivation. Ici encore donc, Merleau-Ponty maintient que nous sommes en droit d'affirmer que la cognition algébrique est le corrélat d'une exploration sensorimotrice dans la mesure où la configuration particulière d'un ensemble de signes algébriques contribue à leur signification mathématique.

Par conséquent, Merleau-Ponty maintient que nous ne pensons véritablement en mathématiques, et il n'y a de développement des mathématiques en tant que discipline, qu'en modifiant la manière dont les

${ }^{44}$ M. Merleau-Ponty, L'institution, la passivité. Notes de course au Collège de France (19541955), Belin, 2003, 96. 
structures mathématiques relativement générales héritées de la tradition sont concrètement structurées dans notre champ d'expérience. Dans cette optique, la compréhension géométrique et algébrique résulte d'une exploration sensori-motrice spécifique du champ phénoménal corrélatif à un espace géométrique ou à un système algébrique donnés.

Ici encore, la désintégration pathologique de l'intentionnalité motrice de Schneider offre un contraste instructif avec l'argument positif de Merleau-Ponty. Alors que la pensée productive en mathématiques implique généralement la production d'organisations plus complexes et plus finement structurées d'acquisitions mathématiques antérieures, le cas de Schneider se distingue par le fait que sa capacité à effectuer de telles réorganisations est significativement déficiente en raison de sa blessure corporelle.

Schneider comprenait linguistiquement et intellectuellement ce qu'est un triangle ou un carré, et la relation entre ces significations ne lui échappait pas. Cependant, il ne pouvait pas accéder à des propriétés géométriques autres que celles évidentes des structures géométriques telles qu'elles lui étaient factuellement présentées. Par exemple, Schneider comprenait que les triangles s'inséraient dans les carrés, mais pas si les triangles devaient être d'abord tournés. ${ }^{45}$ De même, Schneider était capable d'additionner, de soustraire, de multiplier ou de diviser, mais seulement en ce qui concerne les objets placés devant lui ; les problèmes plus abstraits, il ne les résolvait qu'à l'aide d'opérations manuelles

45 M. Merleau-Ponty, Phénoménologie de la perception, Gallimard, 1945, 165. telles que le comptage des doigts. ${ }^{46}$ De même, il ne pouvait pas comprendre, par exemple, que le " double de la moitié » d'un nombre donné est ce nombre même, alors qu'il pouvait effectuer l'opération arithmétique correspondante. ${ }^{47}$

En tant qu'inversion pathologique, le cas de Schneider donc confirme l'affirmation de Merleau-Ponty selon laquelle le sujet de la géométrie et de l'arithmétique est un sujet moteur incarné : une diminution de la capacité à articuler phénoménalement les objets par l'intentionnalité motrice, causée par une lésion cérébrale, est en corrélation avec la diminution de la capacité de Schneider à comprendre les relations géométriques, arithmétiques et algébriques. Cela signifie, en bref, que, tout comme dans le domaine sensorimoteur, la cognition d'ordre supérieur de Schneider a été altérée dans la mesure où sa lésion a limité sa capacité motrice à produire des différenciations comparativement plus complexes de toute structure phénoménale donnée.

\section{Conclusion}

Pour conclure, je résume brièvement ce que je considère comme les aspects les plus intéressants et les plus prometteurs de l'approche phénoménologique de la cognition supérieure incarnée de Merleau-Ponty. Comme je l'ai montré sur les exemples liés aux difficultés neuropathologiques de Schneider et à ses performances compensatoires, MerleauPonty soutient qu'il existe une continuité fondamentale entre les domaines sensorimoteurs et intellectuels de la cognition. Les deux 
domaines doivent être liés à nos capacités corporelles d'articuler les structures phénoménales qui y apparaissent. À la fin des années quarante, l'adoption d'un cadre structuraliste saussurien aide Merleau-Ponty à mieux rendre compte des aspects intersubjectifs et culturels de la cognition, ce qui l'amène à reconnaître qu'il existe également une certaine discontinuité entre la cognition sensorimotrice et la cognition dite supérieure. Certaines "acquisitions " de sens peuvent être conservées même si les fonctions « inférieures » sont désintégrées, comme cela s'est produit dans le cas de Schneider. Cependant, comme on a pu le voir avec l'exemple des mathématiques, Merleau-Ponty soutient que ces structures acquises nécessitent une "reprise " active si elles doivent conserver leur plein sens. Même les structures générales telles que les figures géométriques ou les séries arithmétiques et algébriques nécessitent une reprise active de structuration phénoménale. Ainsi, l'interprétation structurelle dynamique de la cognition de Merleau-Ponty offre une interprétation originale de la relation entre les types de cognition dits inférieurs et supérieurs. Comme je l'ai souligné, MerleauPonty a pu déterminer que le pouvoir articulatoire du langage devait être considéré comme une différenciation plus fine du pouvoir articulatoire que nous trouvons dans l'expérience perceptive sous la forme de l'intentionnalité motrice.

Funding : Work on this study was supported by the project "The Dynamics of Corporeal Intentionality," Palacký University Olomouc, reg. no. JG_2019_006.

\section{Liste de références :}

A. P. Foultier, « Merleau-Ponty's Encounter with Saussure's Linguistics: Misreading, Reinterpretation or Prolongation? ", Chiasmi International, 15 / 2013, 129-150.

A. Gelb \& K. Goldstein, Psychologische Analysen hirnpathologischer Fälle. Johann Ambrosius Barth, 1920.

G. Goldenberg, « Goldstein and Gelb's Case Schn.: A classic case in neuropsychology? ", in Ch. Code, C.-W. Wallesch, Y. Joanette, A. R. Lecours (eds), Classical Cases in Neuropsychology, vol. II, Psychology Press, 2012, 281-299.

K. Godstein, Language and language disturbances; aphasic symptom complexes and their significance for medicine and theory of language, Grune and Stratton, 1948.

A. A. Grünbaum, "Aphasie und Motorik », in Zeitschrift für die gesamte Neurologie und Psychiatrie, 130(1) / 1930, 385-412.

A. Gurwitsch, « Some Aspects and Developments of Gestalt Psychology », in F. Kersten (Ed.), The Collected Works of Aron Gurwitsch (1901-1973): Volume II: Studies in Phenomenology and Psychology. Springer Science \& Business Media, 2009, 1-61.

L. Hass, Merleau-Ponty's Philosophy, Indiana University Press, 2008.

M. Hass \& L. Hass, L., " Merleau-Ponty and the Origin of Geometry » in F. Evans (Ed.), Chiasms: Merleau-Ponty's Notion of Flesh, SUNY Press, 2000, 177-187.

M. Hohol, Foundations of Geometric Cognition, Routledge, 2020.

G. B. Jackson, « Maurice Merleau-Ponty's concept of motor intentionality: Unifying two kinds of bodily agency ", European Journal of Philosophy, 26(2) / 2017, 763-779.

R. Jakobson, Kindersprache, Aphasie, und allgemeine Lautgesetze, De Gruyter Mouton, 1969.

R. T. Jensen, "Motor intentionality and the case of Schneider ", in Phenomenology and the Cognitive Sciences, 8(3) / 2009, 371-388. 


\section{LA PAROLE OPERANTE COMME SPECIFICATION DE L'INTENTIONNALITE MOTRICE CHEZ MERLEAU-PONTY}

S. Matherne, « Merleau-Ponty on abstract thought in mathematics and natural science ", in European Journal of Philosophy, 26(2) / 2018, 780797.

M. Merleau-Ponty, La prose du monde, Gallimard, 1969.

M. Merleau-Ponty, Le monde sensible et le monde de l'expression, Metis Presses, 2011.

M. Merleau-Ponty, Le problème de la parole, Metis Presses, 2020.

M. Merleau-Ponty, L'institution, la passivité. Notes de course au Collège de France (1954-1955), Belin, 2003.

M. Merleau-Ponty, Phénoménologie de la perception, Gallimard, 1945.

M. Merleau-Ponty, Résumés de cours, Gallimard, 1968.

M. Merleau-Ponty, Structure du comportement, P.U.F., 1942.

T. Mooney, «Plasticity, motor intentionality and concrete movement in Merleau-Ponty ", Continental Philosophy Review, 44(4) / 2011, 359381.

K. Romdenh-Romluc, "Science in Merleau-Ponty's phenomenology: From the early work to the later philosophy ", in D. Zahavi (Ed.), Oxford Handbook of the History of Phenomenology, Oxford University Press, 340-359.
F. de Saussure, Cours de linguistique générale, Payot, 1971.

B. Stawarska, Saussure's Philosophy of Language as Phenomenology: Undoing the Doctrine of the Course in General Linguistics, Oxford University Press, 2015.

B. Stawarska, Saussure's Linguistics, Structuralism, and Phenomenology, Palgrave Macmillan, 2020.

B. Stawarska, « Uncanny Errors, Productive Contresens. Merleau-Ponty's Phenomenological Appropriation of F. de Saussure's General Linguistics ", in Chiasmi International, 15 / 2013, 151-165.

M. Wertheimer, "The Syllogism and Productive Thinking ", in W. D. Ellis (Ed.), A Source Book of Gestalt Psychology, Kegan Paul, Trench, Trubner \& Company, 1938, 274-282.

M. Wertheimer, Productive Thinking, Birkhäuser, 2020. 
